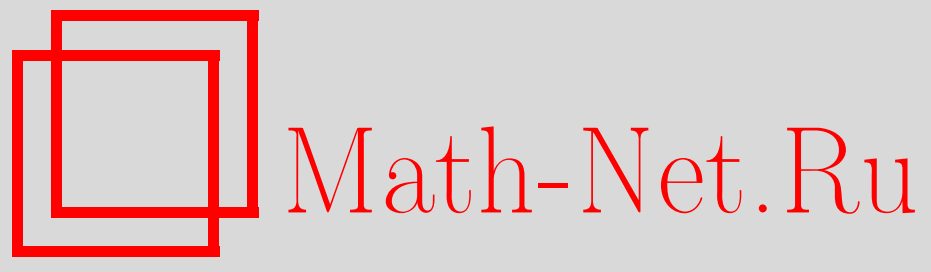

И. А. Дынников, О новой дискретизации комплексного анализа, УМН, 2015, том 70, выпуск 6, 63-84

DOI: https://doi.org/10.4213/rm9687

Использование Общероссийского математического портала Math-Net.Ru подразумевает, что вы прочитали и согласны с пользовательским соглашением http: //www . mathnet.ru/rus/agreement

Параметры загрузки:

IP : 3.85 .183 .62

26 апреля 2023 г., 11:06:58

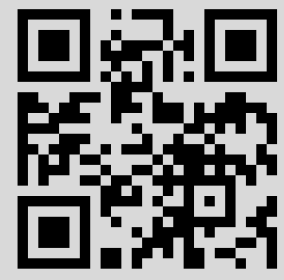




\section{О новой дискретизации комплексного анализа}

\section{И. А. Дынников}

В работе развивается подход к дискретизации комплексного анализа, предложенный С. П. Новиковым и автором в 2003 г. В этом подходе дискретные аналитические функции являются вещественнозначными. В данной работе показано, что для большого класса таких функций на решетке канонически определяется оператор умножения на мнимую единицу. Рассматриваются случаи произвольных решеток для треугольной дискретизации и ромбических решеток - для четырехугольной.

Библиография: 24 названия.

Ключевые слова: дискретные аналитические функции, дискретные голоморфные функции, дискретизация комплексного анализа.

DOI: $10.4213 / \mathrm{rm} 9687$

\section{СоДЕРЖАниЕ}

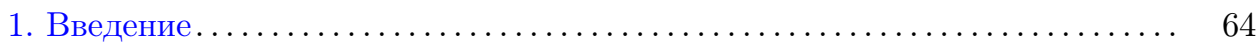

1.1. Классический подход к дискретизации условий Коши-Римана. . 64

1.2. Новый подход к дискретизации ..................... 65

1.3. Общие треугольная и четырехугольная дискретизации условий

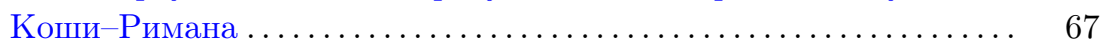

2. Дискретизация на правильной треугольной решетке $\ldots \ldots \ldots \ldots \ldots \ldots \ldots .68$

2.1. Дискретные аналитические функции и многочлены ........... 68

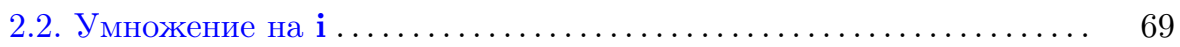

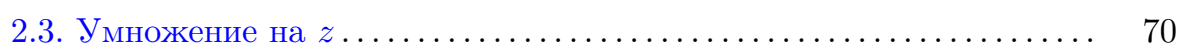

2.4. Базис в пространстве многочленов и экспонента ............ 71

2.5. Формула Коши и обобщенная теорема Лиувилля............ 73

3. Треугольная дискретизация на произвольной решетке ........... 73

4. Четырехугольная дискретизация на ромбической решетке . . . . . . . . 77

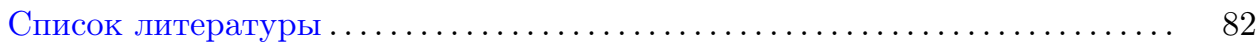

Исследование выполнено за счет гранта Российского научного фонда (проект № 14-50-00005).

(C) И. А. Дынников, 2015 


\section{1. Введение}

В настоящей работе рассматриваются дискретные аналоги аналитических функций на комплексной плоскости $\mathbb{C}$, определенные на двумерной решетке $\mathbb{L} \cong \mathbb{Z}^{2}, \mathbb{L} \subset \mathbb{C}$. Развивается подход, предложенный С. П. Новиковым и автором в 2003 г. в связи с дискретизацией теории солитонов [1]-[5].

Через $m, n \in \mathbb{Z}$ обозначаются координаты на решетке, через $T_{1}, T_{2}$ - базисные сдвиги вдоль решетки:

$$
\left(T_{1} f\right)(m, n)=f(m+1, n), \quad\left(T_{2} f\right)(m, n)=f(m, n+1) .
$$

\section{1. Классический подход к дискретизации условий Коши-Рима-} на. Наиболее известным дискретным аналогом условий Коши-Римана является следующее разностное уравнение, предложенное Ж. Ферранд [6] в 1944 г.:

$$
f(m, n)+\mathbf{i} f(m+1, n)-f(m+1, n+1)-\mathbf{i} f(m, n+1)=0
$$

(здесь $\mathbf{i}$ - мнимая единица), или, что равносильно,

$$
\frac{f(m, n+1)-f(m+1, n)}{\mathbf{i}-1}=\frac{f(m+1, n+1)-f(m, n)}{\mathbf{i}+1} .
$$

Из второй формы записи видно, что условие состоит в равенстве разностных производных по взаимно перпендикулярным направлениям $\mathbf{i}+1$ и $\mathbf{i}-1$. Здесь предполагается, что $\mathbb{L}$ - правильная квадратная решетка гауссовых целых, $(m, n)=m+\mathbf{i} n$.

Уравнение, эквивалентное (1), появлялось в более ранней работе Р. Айзекса [7], которая в годы войны не была широко известна. В ней Айзекс дает также другое определение дискретного аналога условий Коши-Римана:

$$
f(m, n+1)-f(m, n)=\mathbf{i}(f(m+1, n)-f(m, n)) .
$$

Смысл этого уравнения, как и предыдущего, - в равенстве разностных производных по двум перпендикулярным направлениям, но теперь в каждом соотношении участвуют три точки решетки, в отличие от (1), где точек четыре.

Сам Айзекс больше интересовался дискретизацией (2), вводя для нее аналоги многочленов и экспоненты. Теория функций (1) была значительно развита Р. Даффином [8], который изучал для них аналоги комплексных многочленов, а также операции, которые могли бы служить заменой операции произведения аналитических функций.

Следует отметить, что ни для одной дискретизации теории комплексно-аналитических функций не удалось построить полноценного аналога операции произведения двух дискретных аналитических функций, что является главным недостающим звеном этих теорий.

Побочным эффектом в случае обеих дискретизаций (1) и (2) является "удвоение размерности" - пространство дискретных аналитических функций оказывается "вдвое больше", чем можно было бы ожидать исходя из непрерывного аналога. Формально это выражается, например, в том, что пространство дискретных аналитических функций, растущих не быстрее многочлена степени $k$, 
которое естественно принять за аналог многочленов степени $\leqslant k$, имеет комплексную размерность $2 k+2$, а не $k+1$. Кроме того, на пространстве всех дискретных аналитических функций появляется антилинейная инволюция, аналога которой нет в непрерывном случае. Для дискретизации (1) эта инволюция имеет вид $f(m, n) \mapsto(-1)^{m+n} \overline{f(m, n)}$, а для $(2)-$ вид $f(m, n) \mapsto \mathbf{i}^{m-n} \overline{f(m, n)}$.

На сегодняшний день дискретизация (1) находит много приложений для аппроксимации аналитических функций, а также в теории вероятностей и математической физике [9], [10]. Она обобщена в [11] на ромбические решетки (т.е. такие, в которых диагонали базисной ячейки ортогональны), а в более поздних работах [9], [12]-[16] на изорадиальные графы и римановы поверхности, для чего последние разбиваются на четырехугольники. Отметим, что впервые комбинаторика таких разбиений изучалась в работах Н. П. Долбилина, М. И. Штогрина и М. А. Штанько [17], [18] в связи с задачами математической физики.

Отметим также, что теория дискретных гармонических функций, которая тесно связана с дискретизацией (1), изучалась, начиная с работы Люстерника [19] в связи с приложениями к решению задачи Дирихле.

В настоящей работе мы совсем не касаемся принципиально иного, "нелинейного", подхода к дискретизации комплексного анализа, связанного с упаковками кругов и предложенного У. Тёрстоном в [20]. Обзор теорий такого типа см. в $[21]$.

1.2. Новый подход к дискретизации. В работах [2], [3] С. П. Новиков обратил внимание на то, что для построения дискретного аналога преобразования Лапласа естественнее перейти от квадратной к правильной треугольной решетке, т. е. решетке, базисные сдвиги которой происходят вдоль векторов сторон правильного треугольника.

Эти идеи развивались в работах [4], [5], где изучались обобщения на более высокие размерности и симплициальные комплексы и было введено понятие дискретной аффинной связности. Оно связано с решением уравнений вида $(Q \psi)(p)=\sum_{p \in \sigma} a_{\sigma, p} \psi(p)=0$, где $\psi-$ функция на вершинах симплициального комплекса, $a_{\sigma, p}-$ набор коэффициентов связности, ассоциированных с парами $(\sigma, p)$, в которых $\sigma$ - симплекс старшей размерности, а $p$ - его вершина.

В [1] обнаружено, что возникающие в этой теории операторы для триангулированных поверхностей обладают рядом свойств, аналогичных свойствам операторов комплексного дифференцирования $\partial / \partial z$ и $\partial / \partial \bar{z}$.

А именно, пусть $M$ - произвольная ориентированная поверхность с фиксированной триангуляцией $T$, которая обладает раскраской вершин в три цвета такой, что никакие две вершины одного цвета не соединены ребром. Треугольники такой триангуляции допускают шахматную раскраску. Пусть такая раскраска фиксирована.

Обозначим через $V$ пространство вещественнозначных функций на множестве вершин триангуляции $T$, через $W$ пространство функций на множестве белых треугольников и через $B$ пространство функций на множестве черных треугольников. 
Определим операторы $Q_{\mathrm{w}}: V \rightarrow W$ и $Q_{\mathrm{b}}: V \rightarrow B$ следующим образом. Для белого треугольника $t$ с вершинами $p_{1}, p_{2}, p_{3}$ положим $\left(Q_{\mathrm{w}} \psi\right)(t)=\psi\left(p_{1}\right)+$ $\psi\left(p_{2}\right)+\psi\left(p_{3}\right)$, и аналогично для черных треугольников и оператора $Q_{\mathrm{b}}$.

Как показано в [1], пара операторов $Q_{\mathrm{w}}$ и $Q_{\mathrm{b}}$ обладает рядом общих свойств с операторами $\partial / \partial z$ и $\partial / \bar{z}$. Мы коротко остановимся на этих свойствах.

Теорема 1. Если поверхность $M$ связна и замкнута, то ядро каждого из операторов $Q_{\mathrm{w}}$ и $Q_{\mathrm{b}}$ двумерно, причем $\operatorname{ker} Q_{\mathrm{w}}=\operatorname{ker} Q_{\mathrm{b}}$. Пересечение $\operatorname{ker} Q_{\mathrm{w}} \cap$ $\operatorname{ker} Q_{\mathrm{b}}$ двумерно для любой связной поверхности $M$.

Таким образом, пересечение $H_{0}$ ядер операторов $Q_{\mathrm{w}}$ и $Q_{\mathrm{b}}$ служит аналогом пространства постоянных функций и совпадает с каждым из ядер, если поверхность замкнута. Пространство $H=\operatorname{ker} Q_{\mathrm{b}}$ мы считаем аналогом пространства голоморфных функций на $M$.

В случае компактных поверхностей с краем имеет место аналог принципа максимума. Чтобы его сформулировать, введем отображение $\psi \mapsto \widehat{\psi}$, которое "голоморфной" функции $\psi \in H$ ставит в соответствие отображение $\widehat{\psi}$ из множества черных треугольников в двумерное пространство "констант" $H_{0}$ следующим образом. Если $t$ - черный треугольник, то $\widehat{\psi}(t)$ есть функция из $H_{0}$, которая на вершинах треугольника $t$ совпадает с $\psi$ (она единственна).

Теорема 2 (принцип максимума). Для любой функиии $\psi \in H$ образы всех черных треугольников при отображении $\widehat{\psi}$ содержатся в выпуклой оболочке образов черных треугольников, имеющих непустое пересечение с границей $\partial M$.

Если $M$ - это плоскость $\mathbb{R}^{2}$, разбитая на правильные треугольники, то пространства $V, W$ и $B$ можно отождествить так, что операторы $Q_{\mathrm{b}}$ и $Q_{\mathrm{w}}$ становятся взаимно сопряженными друг другу и действуют из $V$ в $V$. При этом множество вершин есть правильная треугольная решетка, и операторы $Q_{\mathrm{b}}$ и $Q_{\mathrm{w}}$ можно записать через ее базисные сдвиги $T_{1}, T_{2}$ :

$$
Q_{\mathrm{b}}=1+T_{1}+T_{2}, \quad Q_{\mathrm{w}}=Q_{\mathrm{b}}^{+}=1+T_{1}^{-1}+T_{2}^{-1} .
$$

Считая эти операторы аналогами $\partial / \partial \bar{z}$ и $\partial / \partial z$, естественно определить пространство многочленов степени $\leqslant k$ как множество решений системы

$$
Q_{\mathrm{b}} \psi=0, \quad Q_{\mathrm{w}}^{k+1} \psi=0 .
$$

Подчеркнем, что все коэффициенты операторов и значения функций считаются вещественными. Как следствие, пространство многочленов оказывается "правильной” размерности, однако пространство дискретных аналитических функций оказывается определенным только над $\mathbb{R}$.

Основной результат данной работы состоит во введении на большом подпространстве дискретных аналитических функций комплексной структуры, которая, как показано ниже, определяется однозначно с точностью до сопряжения требованием инвариантности относительно группы сдвигов решетки.

Кроме того, ниже обсуждаются другие известные фундаментальные свойства данной дискретизации, а также их обобщения на случай произвольных решеток. 
ЗАмечАниЕ 1. В работе [22] ставится задача нахождения дискретного аналога функций $z^{n}$ на плоскости Лобачевского, разбитой на правильные треугольники с углом $\pi / k$ при вершине, $k \geqslant 4$. В этом случае ситуация принципиально отличается от случая евклидовой плоскости: множество вершин триангуляции не образует решетку $\mathbb{Z}^{2}$, а аналогами $z^{n}$ должны быть ограниченные функции. В настоящий момент данный вопрос не решен.

\section{3. Общие треугольная и четырехугольная дискретизации усло-} вий Коши-Римана. Введем обобщения разностных уравнений (1) и (2) на случай произвольной, не обязательно квадратной, решетки.

Пусть $\mathbb{L}$ - решетка в $\mathbb{C}$ с фиксированным базисом $\tau_{1}, \tau_{2}$. Точка $(m, n) \in \mathbb{L}$ имеет комплексную координату

$$
z(m, n)=m \tau_{1}+n \tau_{2} .
$$

Для нас будет играть роль только отношение $\tau_{2} / \tau_{1}$, которое мы будем называть параметром решетки и обозначать через $\tau$.

Уравнения (1) и (2) естественно обобщаются следующим образом.

Треугольной дискретизаиией условий Коши-Римана называется разностное уравнение

$$
\frac{f(m+1, n)-f(m, n)}{z(m+1, n)-z(m, n)}=\frac{f(m, n+1)-f(m, n)}{z(m, n+1)-z(m, n)},
$$

или, что эквивалентно,

$$
f(m, n)-\frac{\tau}{\tau-1} f(m+1, n)+\frac{1}{\tau-1} f(m, n+1)=0 .
$$

Под четырехугольной дискретизацией условий Коши-Римана мы понимаем следующее уравнение:

$$
\frac{f(m+1, n+1)-f(m, n)}{z(m+1, n+1)-z(m, n)}=\frac{f(m, n+1)-f(m+1, n)}{z(m, n+1)-z(m+1, n)},
$$

или, что эквивалентно,

$$
f(m+1, n+1)-f(m, n)-\frac{\tau+1}{\tau-1}(f(m, n+1)-f(m+1, n))=0 .
$$

Ниже мы рассмотрим всевозможные треугольные дискретизации и четырехугольные дискретизации для ромбических решеток. В этих случаях калибровочным преобразованием можно сделать все коэффициенты вещественными. Дискретные аналоги аналитических функций тогда естественно определить как вещественнозначные решения соответствующих уравнений. Для случая правильной треугольной решетки мы, разумеется, получим упомянутую выше конструкцию из работы [1].

Отметим, что вещественная форма для дискретного оператора Коши-Римана появляется в работе Даффина [8], но не используется там для определения дискретных аналитических функций как вещественнозначных в соответствующей калибровке. 


\section{2. Дискретизация на правильной треугольной решетке}

2.1. Дискретные аналитические функции и многочлены. Итак, пусть сначала $\mathbb{L}_{\triangle} \cong \mathbb{Z}^{2}$ - правильная треугольная решетка в $\mathbb{C}$, т. е. решетка с параметром $\tau=(1+\mathbf{i} \sqrt{3}) / 2$. Треугольная дискретизация условий Коши-Римана (3) превращается в уравнение

$$
f(m, n)+\frac{-1+\mathbf{i} \sqrt{3}}{2} f(m+1, n)+\frac{-1-\mathbf{i} \sqrt{3}}{2} f(m, n+1)=0 .
$$

Калибровочным преобразованием

$$
f(m, n) \mapsto \widetilde{f}(m, n)=\left(\frac{-1+\mathbf{i} \sqrt{3}}{2}\right)^{m-n} f(m, n)
$$

оно приводится к уравнению с вещественными коэффициентами:

$$
\tilde{f}(m, n)+\widetilde{f}(m+1, n)+\widetilde{f}(m, n+1)=0,
$$

которое и положено в основу определения дискретной аналитической функции в работе [1].

ОПРЕДЕЛЕНИЕ 1. Дискретной аналитической (голоморфной) функцией треугольного типа на $\mathbb{L}_{\triangle}$ называются вещественнозначные решения $\widetilde{f}$ разностного уравнения (6).

В дальнейшем мы опускаем слова “треугольного типа", так как в этом разделе рассматривается только треугольная дискретизация.

Таким образом, роль оператора Коши-Римана $\partial / \partial \bar{z}$ играет оператор

$$
Q=1+T_{1}+T_{2}
$$

Пространство $\operatorname{ker} Q$ всех дискретных аналитических функций будем обозначать через $\mathscr{A}$.

В качестве аналога оператора $\partial / \partial z$ (точнее, $-\partial / \partial z$, но это здесь не играет роли) естественно было бы взять формально сопряженный оператор $Q^{+}=$ $1+T_{1}^{-1}+T_{2}^{-1}$. Однако по ряду причин здесь нам будет удобнее вместо него работать с оператором

$$
D=T_{1} T_{2} Q^{+}=T_{1}+T_{2}+T_{1} T_{2},
$$

степени которого имеют то же ядро, что и степени оператора $Q^{+}$. Классы функций, которые мы будем рассматривать, при этой замене сохраняются. Взяв $D$ в качестве аналога $\partial / \partial z$, естественно дать следующее определение.

ОПРЕДЕЛЕНИЕ 2. Дискретным голоморфным многочленом (треуголъного muпа) степени $k$ на решетке $\mathbb{L}_{\triangle}$ называется дискретная голоморфная функция $f$, для которой

$$
D^{k+1} f=0, \quad D^{k} f \neq 0 .
$$

Это определение введено в работе [1], где доказано следующее утверждение.

ПРеДЛОЖениЕ 1. Пространство $\mathscr{P}_{k}$ всех дискретных голоморфных многочленов степени не выше $k$ имеет над $\mathbb{R}$ размерность $2 k+2$.

Заметим, что $\mathscr{P}_{k}$ определено пока только как вещественное пространство. 


\section{2. Умножение на i.}

Теорема 3. На пространстве $\mathscr{P}=\bigcup_{k=0}^{\infty} \mathscr{P}_{k}$ всех дискретных голоморфных многочленов существует оператор $\mathfrak{I}$, коммутирующий со сдвигами $T_{1}, T_{2}$, квадрат которого равен -1. Такой оператор единственен с точностью до знака.

ДокАзАтельство. Сначала докажем существование. Имеет место тождество

$$
\left(T_{2}-T_{1}\right)^{2}=\left(T_{1}+T_{2}+3\right) Q-3-4 D .
$$

Для ограничения на пространство $\mathscr{P}$ получаем:

$$
\left.\left(T_{2}-T_{1}\right)^{2}\right|_{\mathscr{P}}=-\left.3\left(1+\frac{4}{3} D\right)\right|_{\mathscr{P}} .
$$

Оператор $D$ нильпотентен на каждом подпространстве $\mathscr{P}_{k}$, поэтому можно определить оператор $\left.(1+(4 / 3) D)^{-1 / 2}\right|_{\mathscr{P}_{k}}$, разложив это выражение в формальный ряд по $D$ и отбросив члены порядка выше $k$. Тогда оператор

$$
\mathfrak{I}=\left.\frac{1}{\sqrt{3}}\left(T_{2}-T_{1}\right)\left(1+\frac{4}{3} D\right)^{-1 / 2}\right|_{\mathscr{P}}
$$

обладает нужными свойствами. Действительно, на каждом подпространстве $\mathscr{P}_{k}$ его можно записать как разностный оператор с постоянными коэффициентами, поэтому он коммутирует с базисными сдвигами. По построению $\mathfrak{I}^{2}=-1$.

Теперь докажем единственность. Для этого будем рассматривать Я как комплексное векторное пространство с оператором (7) в качестве комплексной структуры. Пусть $\mathfrak{I}^{\prime}$ - другой оператор, удовлетворяющий условиям теоремы.

Так как $\mathfrak{I}^{\prime}$ коммутирует с базисными сдвигами, он коммутирует также с $\mathfrak{I}$, т. е. является линейным оператором над $\mathbb{C}$. Из равенства $\mathfrak{I}^{\prime 2}=-1$ следует, что все пространство $\mathscr{P}$ распадается в прямую сумму двух собственных подпространств, отвечающих собственным значениям i и $-\mathbf{i}$. Нам остается показать, что одно из этих подпространств нулевое, а другое - все $\mathscr{P}$.

Оператор $\mathfrak{I}^{\prime}$ коммутирует с $D$, поэтому каждое собственное подпространство для $\mathfrak{I}^{\prime}$ инвариантно относительно $D$. Поскольку оператор $D$ понижает степень многочлена на единицу, то в любом нетривиальном собственном подпространстве оператора $\mathfrak{I}^{\prime}$ должен найтись ненулевой вектор из $\mathscr{P}_{0}=\operatorname{ker} D$. Но ядро $\operatorname{ker} D$ двумерно над $\mathbb{R}$ и, следовательно, одномерно над $\mathbb{C}$. Теорема доказана.

В дальнейшем мы фиксируем оператор (7) в качестве умножения на i в $\mathscr{P}$. Этот оператор "нелокальный”, но для каждого $k$ ограничение $\mathfrak{I}$ на подпространство $\mathscr{P}_{k}$ можно записать как разностный оператор с постоянными коэффициентами. Например,

$\left.\mathfrak{I}\right|_{\mathscr{P}_{0}}=\left.\frac{1}{\sqrt{3}}\left(T_{2}-T_{1}\right)\right|_{\mathscr{P}},\left.\quad \mathfrak{I}\right|_{\mathscr{P}_{1}}=\frac{1}{3 \sqrt{3}}\left(T_{2}-T_{1}+T_{1} T_{2}^{-1}-T_{2}^{-1}+T_{1}^{-1}-T_{1}^{-1} T_{2}\right)$.

В разделе 3 мы значительно расширим область определения оператора $\mathfrak{~}$ 
2.3. Умножение на z. В работе [8] для дискретизации четырехугольного типа на квадратной решетке Даффин вводит оператор "умножения на $z$ ", действующий в пространстве всех дискретных аналитических функций. Такой оператор есть и в нашем случае, он имеет вид

$$
Z=m T_{2}-n T_{1}, \quad(Z f)(m, n)=m f(m, n+1)-n f(m+1, n) .
$$

ПредЛОжение 2. (а) Оператор $Z$ сохраняет пространство $\mathscr{A}$ дискретных аналитических функиий на $\mathbb{L}_{\triangle}$.

(b) Если $f$-дискретный голоморфный многочлен, то $Z f$ - также дискретный голоморфный многочлен, причем $\operatorname{deg}(Z f)=\operatorname{deg} f+1$.

(c) Onератор $Z$ в пространстве дискретных голоморбных многочленов $\mathscr{P}$ линеен над $\mathbb{C}$.

ДокАзАтЕльство. (а) Утверждение следует из того, что $Z$ коммутирует с $Q$, что легко проверяется.

(b) Простая проверка дает

$$
[D, Z]=T_{1} T_{2}\left(T_{2}-T_{1}\right)=(1+D-Q)\left(T_{2}-T_{1}\right) .
$$

Это оператор с постоянными коэффициентами, следовательно, он сохраняет подпространство $\mathscr{P}$ и может быть там записан в виде

$$
\left.[D, Z]\right|_{\mathscr{P}}=\left.\sqrt{3} \Im(1+D)\left(1+\frac{4}{3} D\right)^{1 / 2}\right|_{\mathscr{P}} .
$$

Положим

$$
\widetilde{Z}=-\left.\frac{1}{\sqrt{3}} Z \mathfrak{I}(1+D)^{-1}\left(1+\frac{4}{3} D\right)^{-1 / 2}\right|_{\mathscr{P}} .
$$

Этот оператор определен на $\mathscr{P}$, но мы пока не доказали, что его образ содержится в $\mathscr{P}$. Имеет место соотношение

$$
\left.[D, \widetilde{Z}]\right|_{\mathscr{P}}=1 .
$$

Возьмем произвольный базис $f_{0}, g_{0} \in \mathscr{P}_{0}$ над $\mathbb{R}$ и положим

$$
f_{k+1}=\widetilde{Z} f_{k}, \quad g_{k+1}=\widetilde{Z} g_{k}, \quad k=0,1,2, \ldots
$$

Из (9) по индукции получаем

$$
D f_{k}=k f_{k-1}, \quad k=0,1,2, \ldots,
$$

откуда следуют соотношения $f_{k}, g_{k} \in \mathscr{P}_{k}, \operatorname{deg} f_{k}=\operatorname{deg} g_{k}=k$, а также линейная независимость всех функций $f_{k}, g_{k}, k=0,1,2, \ldots$ Из соображений размерности функции $f_{0}, g_{0}, \ldots, f_{k}, g_{k}$ образуют базис в $\mathscr{P}_{k}$, и, значит, $\widetilde{Z} \mathscr{P}_{k} \subset \mathscr{P}_{k+1}$, причем $\widetilde{Z}\left(\mathscr{P}_{k} \backslash \mathscr{P}_{k-1}\right) \subset \mathscr{P}_{k+1} \backslash \mathscr{P}_{k}$. Оператор $\left.\left(\mathfrak{I}(1+D)^{-1}(1+(4 / 3) D)^{-1 / 2}\right)\right|_{\mathscr{P}_{k}}$ является изоморфизмом для каждого $k$, откуда следует искомое утверждение о $Z$. 
(с) Для доказательства нужно проверить, что $Z$ коммутирует с I. Мы уже знаем, что $Z$ сохраняет $\mathscr{P}$, поэтому можем пользоваться формализмом степенных рядов по $D$.

Поскольку оператор $[D, Z]$ имеет постоянные коэффициенты, из (8) получаем:

$$
\begin{aligned}
{[\mathfrak{I}, Z]=} & {\left.\left[\frac{1}{\sqrt{3}}\left(T_{2}-T_{1}\right)\left(1+\frac{4}{3} D\right)^{-1 / 2}, Z\right]\right|_{\mathscr{P}} } \\
= & \left.\frac{1}{\sqrt{3}}\left(\left[T_{2}-T_{1}, Z\right]\left(1+\frac{4}{3} D\right)^{-1 / 2}-\frac{2}{3}\left(T_{2}-T_{1}\right)\left(1+\frac{4}{3} D\right)^{-3 / 2}[D, Z]\right)\right|_{\mathscr{P}} \\
= & \frac{1}{\sqrt{3}}\left(-2 T_{1} T_{2}\left(1+\frac{4}{3} D\right)^{-1 / 2}\right. \\
& \left.\left.\left.-\frac{2}{3}\left(T_{2}-T_{1}\right)\left(1+\frac{4}{3} D\right)^{-3 / 2} T_{1} T_{2}\left(T_{2}-T_{1}\right)\right)\left.\right|_{\mathscr{P}}\right)^{-1 / 2}-2\left(1+\frac{4}{3} D\right)^{-1 / 2} T_{1} T_{2} \mathfrak{I}^{2}\right)\left.\right|_{\mathscr{P}}=0 .
\end{aligned}
$$

Предложение доказано.

Отметим, что оператор комплексной структуры $\mathfrak{I}$ определен канонически (с точностью до знака) простым требованием коммутирования с базисными сдвигами, но задан пока только на пространстве дискретных голоморфных многочленов. Операторы же $D$ и $Z$ определены для всех дискретных голоморфных функций, но выбор их не является каноническим и не выдерживает, например, поворота плоскости на $2 \pi / 3$, сохраняющего решетку $\mathbb{L}_{\triangle}$. Тем не менее пространства дискретных аналитических функций и многочленов при таком повороте сохраняются.

2.4. Базис в пространстве многочленов и экспонента. Существует несколько способов строить дискретный аналог базиса $1, z, z^{2}, \ldots$ в пространстве многочленов. К сожалению, различные “естественные" подходы к построению такого аналога выдают разные ответы.

Даффин в [8] строит "псевдостепени" $z^{(i)}$ для дискретизации на квадратной решетке, исходя из аналога интегрирования вдоль пути. Функция $z^{(k+1)}$ получается из $z^{(k)}$ взятием “первообразной” (и соответствующей нормировкой). При этом операция взятия первообразной не допускает однозначно определенной обратной операции - аналога дифференцирования $\partial / \partial z$. “Дифференцирование" в этой теории имеет ту же степень неоднозначности, что и взятие первообразной.

В работе [1] в пространстве дискретных голоморфных многочленов вводится базис, исходя из соображения, что аналог $z^{n}$ должен иметь "нуль большой кратности", которым является большой треугольник, целиком заполненный нулями. При этом не удается сделать этот треугольник симметричным относительно поворотов решетки на $2 \pi / 3$, а также написать явные общие формулы.

Для целей настоящей работы мы воспользуемся самым простым и в то же время универсальным рецептом получения явных формул для базиса в пространстве $\mathscr{P}$, заимствованным из [23] (некоторые элементы метода есть уже в работах Айзекса и Даффина для квадратных дискретизаций). 
Определим аналитические функции $\lambda(\kappa)$ и $\mu(\kappa)$ в круге $|\kappa|<3 / 4$ из уравнений

$$
1+\lambda(\kappa)+\mu(\kappa)=0, \quad \lambda(\kappa)+\mu(\kappa)+\lambda(\kappa) \mu(\kappa)=\kappa, \quad \lambda(0)=e^{2 \pi \mathbf{i} / 3} .
$$

Явный вид их таков:

$$
\lambda(\kappa)=-\frac{1}{2}+\frac{\sqrt{3} \mathbf{i}}{2} \sqrt{1+\frac{4}{3} \kappa}, \quad \mu(\kappa)=-\frac{1}{2}-\frac{\sqrt{3} \mathbf{i}}{2} \sqrt{1+\frac{4}{3} \kappa} .
$$

Положим $E(m, n ; \kappa)=\lambda(\kappa)^{m} \mu(\kappa)^{n}$ и определим вещественнозначные функции $p_{i}, q_{i}, i=0,1,2, \ldots$, на решетке $\mathbb{L}_{\triangle}$, исходя из разложения $E(m, n ; \kappa)$ в ряд по $\kappa$ :

$$
\begin{aligned}
& E(m, n ; \kappa)=\sum_{i=0}^{\infty}\left(p_{i}(m, n)+\mathbf{i} q_{i}(m, n)\right) \frac{\kappa^{i}}{i !}, \\
& p_{i}(m, n)+\mathbf{i} q_{i}(m, n)=\left.\frac{\partial^{i}}{\partial \kappa^{i}} E(m, n ; \kappa)\right|_{\kappa=0} .
\end{aligned}
$$

Легко видеть, что $p_{i}$ и $q_{i}$ являются вещественной и мнимой частями функции вида

$$
P_{i}(m, n) e^{2 \pi \mathbf{i}(m-n) / 3}
$$

где $P_{i}$ - некоторый многочлен степени $i$ с комплексными коэффициентами.

ПреДЛОЖениЕ 3. Функиии $p_{i}, q_{i}, i=0,1,2, \ldots$, образуют базис над $\mathbb{R}$ пространства Я дискретных голоморфных многочленов. Операторы $D$ и $\mathfrak{I}$ записъваются в этом базисе следующим образом:

$$
D p_{i}=i p_{i-1}, \quad D q_{i}=i q_{i-1}, \quad \Im p_{i}=q_{i}, \quad \Im q_{i}=-p_{i} .
$$

ДокАЗАТЕЛЬСтво. По построению имеем

$$
T_{1} E(m, n ; \kappa)=\lambda(\kappa) E(m, n ; \kappa), \quad T_{2} E(m, n ; \kappa)=\mu(\kappa) E(m, n ; \kappa),
$$

откуда следует, что

$$
(Q E)(m, n ; \kappa)=0, \quad(D E)(m, n ; \kappa)=\kappa E(m, n ; \kappa)
$$

Это означает, что функции $p_{i}, q_{i}$ дискретные голоморфные, а оператор $D$ действует на них так, как указано в (10).

Рассматривая функцию $E(m, n ; \kappa)$ как ряд по $\kappa$ с коэффициентами в $\mathscr{P}$, можно применить к ней оператор I:

$$
(\Im E)(m, n ; \kappa)=\frac{1}{\sqrt{3}}(\mu(\kappa)-\lambda(\kappa))\left(1+\frac{4}{3} \kappa\right)^{-1 / 2} E(m, n ; \kappa)=-\mathbf{i} E(m, n ; \kappa) .
$$

Отсюда вытекают два последних равенства в (10). Предложение доказано. 
2.5. Формула Коши и обобщенная теорема Лиувилля. В дискретном случае имеет место полный аналог следующей обобщенной теоремы Лиувилля.

Теорема 4. Если дискретная голоморфная функиия $f$ растет не быстрее многочлена степени $k$, m.е. $|f(m, n)|<C_{1}+C_{2}\left(m^{2}+n^{2}\right)^{k / 2}$, где $C_{1} u C_{2}$ - некоторые константы, то она является дискретным голоморфным многочленом степени $\leqslant k$.

Доказательство также полностью аналогично непрерывному случаю, достаточно доказать аналог интегральной формулы Коши и построить правильный аналог ядра Коши $1 / z$ (функции Грина для $Q$ ). Для четырехугольной дискретизации на квадратной решетке это сделал уже Даффин в [8]. Для треугольной дискретизации на правильной треугольной решетке аналог формулы Коши указан в [1].

ПРЕДЛОЖЕНИЕ 4. Пусть функиия $G: \mathbb{L} \triangle \rightarrow \mathbb{R}$ удовлетворяет уравнению

$$
(Q G)(m, n)= \begin{cases}1, & \text { если }(m, n)=(0,0), \\ 0 & \text { в противном случае. }\end{cases}
$$

Пусть $f: \mathbb{L}_{\triangle} \rightarrow \mathbb{R}-$ дискретная голоморфная функиия, а $\Omega \subset \mathbb{C}-$ некоторая ограниченная область. Тогда для любой внутренней точки $(m, n) \in \Omega$ имеет место равенство

$$
f(m, n)=\sum_{\left(m^{\prime}, n^{\prime}\right) \sim \partial \Omega}(Q \widehat{f})\left(m^{\prime}, n^{\prime}\right) G\left(m-m^{\prime}, n-n^{\prime}\right),
$$

где $\widehat{f}$ обозначает функиию, совпадающую с $f$ внутри $\Omega$ и равную нулю вне $\Omega, a$ запись $\left(m^{\prime}, n^{\prime}\right) \sim \partial \Omega$ означает, что точка $\left(m^{\prime}, n^{\prime}\right)$ расположена возле границь области, а именно так, что треугольник с вершинами $\left(m^{\prime}, n^{\prime}\right),\left(m^{\prime}+1, n^{\prime}\right)$ u $\left(m^{\prime}, n^{\prime}+1\right)$ пересекается с $\partial \Omega$.

П. Г. Гриневич и Р. Г. Новиков в [23] заметили следующее обстоятельство, упущенное в [1], где в качестве $G$ использовалась быстро растущая функция.

ПреДЛОЖЕНИЕ 5. Существует ровно одно ограниченное решение уравнения (11). Оно задается формулой

$$
G(m, n)=\frac{1}{4 \pi^{2}} \int_{0}^{2 \pi} \int_{0}^{2 \pi} \frac{e^{\mathbf{i}(m x+n y)}}{1+e^{\mathbf{i} x}+e^{\mathbf{i} y}} d x d y .
$$

Эта функиия $G$ убывает на бесконечности как $O\left(1 / \sqrt{m^{2}+n^{2}}\right)$. Кроме того, для любого $k=0,1,2, \ldots$ функиия $Q^{k} G$ убьвает на бесконечности как $O\left(\left(m^{2}+n^{2}\right)^{-(k+1) / 2}\right)$.

Отсюда, как указано в [23], следует теорема 4.

\section{3. Треугольная дискретизация на произвольной решетке}

Приведенные выше результаты для правильной треугольной решетки переносятся без принципиальных изменений на произвольную решетку. Мы будем обозначать через $\mathbb{L}^{a b c}$ некоторую решетку, базисный треугольник которой име- 
ет длины сторон $a, b, c$. Разумеется, для нас играют роль только отношения $a / c$ и $b / c$. Углы базисного треугольника, противолежащие сторонам $a, b, c$, обозначим через $\alpha, \beta, \gamma$ соответственно (см. рис. 1 ).

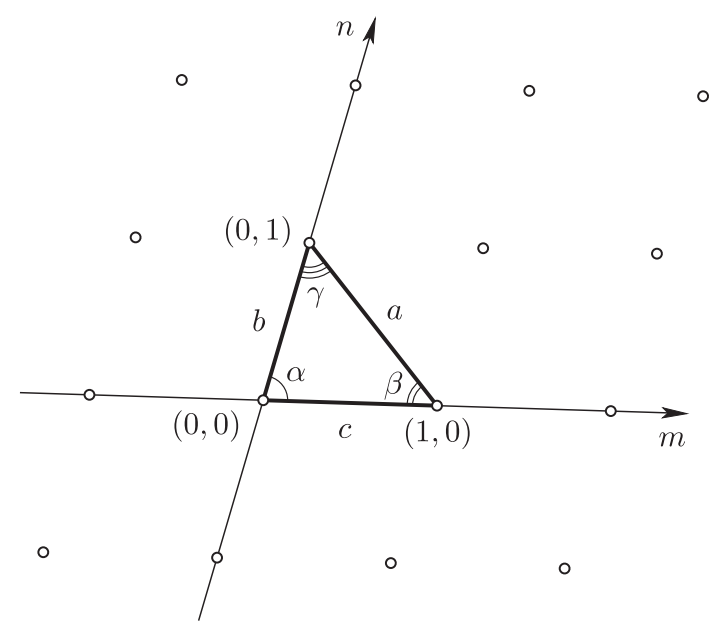

Рис. 1. Решетка $\mathbb{L}^{a b c}$

Треугольная дискретизация (3) условий Коши-Римана выглядит для $\mathbb{L}^{a b c}$ следующим образом:

$$
a f(m, n)-b e^{-\mathbf{i} \gamma} f(m+1, n)-c e^{\mathbf{i} \beta} f(m, n+1)=0 .
$$

После унитарного калибровочного преобразования

$$
f(m, n) \mapsto \widetilde{f}(m, n)=(-1)^{m+n} e^{\mathbf{i}(\beta n-\gamma m)} f(m, n)
$$

получаем уравнение с вещественными коэффициентами:

$$
a \tilde{f}(m, n)+b \tilde{f}(m+1, n)+c \tilde{f}(m, n+1)=0 .
$$

ОПРЕДЕЛЕНИЕ 3. Дискретной аналитической (голоморфной) функцией (треугольного типа) на $\mathbb{L}^{a b c}$ будем называть вещественнозначные решения $\widetilde{f}$ разностного уравнения (14).

Мы используем ту же систему обозначений, что и в предыдущем разделе, добавляя индекс " $a b c$ ", чтобы показать зависимость от геометрии решетки. В частности, $\mathscr{A}^{a b c}$ обозначает пространство дискретных голоморфных функций, а $\mathscr{P}^{a b c} \subset \mathscr{A}^{a b c}$ - подпространство дискретных голоморфных многочленов. Аналоги операторов $\partial / \partial \bar{z}$ и $\partial / \partial z$ в данном случае таковы:

$$
Q^{a b c}=1+\frac{b}{a} T_{1}+\frac{c}{a} T_{2}, \quad D^{a b c}=\frac{c}{a} T_{1}+\frac{b}{a} T_{2}+T_{1} T_{2} .
$$


ПреДлоЖЕнИЕ 6. Для произволъной решетки $\mathbb{L}^{a b c}$ верны все результаты раздела 2 с заменой $D$ на $D^{a b c}, Q$ на $Q^{a b c}$ и т.д. Операторы э и $Z$ обобщаются следующим образом:

$$
\begin{aligned}
\mathfrak{I}^{a b c} & =\left.\frac{1}{\sin \alpha}\left((\cos \gamma) T_{2}-(\cos \beta) T_{1}\right)\left(1+\frac{D^{a b c}}{\sin \beta \sin \gamma}\right)^{-1 / 2}\right|_{\mathscr{P} a b c}, \\
Z^{a b c} & =\frac{c}{a} m T_{2}-\frac{b}{a} n T_{1},
\end{aligned}
$$

а экспоненииальная функиия $E(m, n ; \kappa)$ и функиия Грина $G^{a b c}(m, n)$ для $Q^{a b c}$ обобщаются так:

$$
\begin{aligned}
E^{a b c}(m, n ; \kappa)=( & \left.-\cos \gamma+\mathbf{i} \sin \gamma \sqrt{1+\frac{\kappa}{\sin \beta \sin \gamma}}\right)^{m} \\
& \times\left(-\cos \beta-\mathbf{i} \sin \beta \sqrt{1+\frac{\kappa}{\sin \beta \sin \gamma}}\right)^{n}, \\
G^{a b c}(m, n)= & \frac{1}{4 \pi^{2}} \int_{0}^{2 \pi} \int_{0}^{2 \pi} \frac{e^{\mathbf{i}(m x+n y)}}{1+(b / a) e^{\mathbf{i} x}+(c / a) e^{\mathbf{i} y}} d x d y .
\end{aligned}
$$

Мы опускаем доказательство, которое не содержит ничего содержательно нового по сравнению со случаем правильной треугольной решетки.

Функции сбалансированного роста. Здесь мы снова вернемся к правильной треугольной решетке $\mathbb{L}_{\triangle}=\mathbb{L}^{111}$.

Обозначим через $\Lambda^{u v}$ оператор умножения на функцию $u^{m} v^{n}$ :

$$
\Lambda^{u v} f(m, n)=u^{m} v^{n} f(m, n) .
$$

Легко видеть, что

$$
Q \Lambda^{u v}=\Lambda^{u v} Q^{1 u v}
$$

откуда сразу вытекает следующий результат.

ПреДЛОЖенИЕ 7. Для любъх $a, b, c>0$, удовлетворяющих неравенствам треугольника, ограничение оператора $\Lambda^{u v}$, где $u=b / a, v=c / a$, на $\mathscr{A}^{a b c}$ есть изоморфизм из $\mathscr{A}^{a b c}$ в $\mathscr{A}=\mathscr{A}^{111}$.

Таким образом, пространства дискретных голоморфных функций на решетках с различной геометрией можно отождествить друг с другом. Но при этом пространства дискретных голоморфных многочленов не отождествляются, так как

$$
D \Lambda^{u v} \neq \Lambda^{u v} D^{1 u v}
$$

ПРЕДЛОЖЕНИЕ 8. Пространства $\mathscr{Q}^{u v}=\Lambda^{u v}\left(\mathscr{P}^{1 u v}\right)$ при всевозможных $u, v>0$, для которых тройка $(1, u, v)$ удовлетворяет неравенствам треугольника, образуют прямую сумму в $\mathscr{A}$.

Пусть тройка $(1, u, v)$ удовлетворяет неравенствам треугольника. Функиия $f \in \mathscr{A}$ принадлежит подпространству $\mathscr{Q}^{\text {иv }}$ тогда и только тогда, когда 
найдутся $k \in \mathbb{N}$ и $C_{1}, C_{2} \in \mathbb{R}$ такие, что для всех $(m, n) \in \mathbb{Z}^{2}$ выполнено неравенство

$$
|f(m, n)|<C_{1}+C_{2}\left(m^{2}+n^{2}\right)^{k / 2} u^{m} v^{n} .
$$

(Такие функиии мы будем называть функииями сбалансированного роста.)

Каждое из подпространств $\mathscr{Q}^{\text {иv }}$ инвариантно относительно $D$ и $Z$.

ДокАЗАТЕЛЬство. Первые два утверждения следуют из того, что пространство $\mathscr{P}^{1 u v}$ состоит из всех дискретных голоморфных функций на $\mathbb{L}^{1 u v}$ полиномиального роста. Функции из $\mathscr{Q}^{u v}$ для различных пар $(u, v)$ имеют экспоненциальный рост с различными основаниями, что влечет их независимость.

Инвариантность относительно $D$ следует из того, что $D$ имеет постоянные коэффициенты. Любой оператор с постоянными коэффициентами, очевидно, сохраняет $\mathscr{P}^{1 u v}$ и коммутирует с $\Lambda^{u v}$, а значит, сохраняет и $\mathscr{Q}^{u v}=\Lambda^{u v}\left(\mathscr{P}^{1 u v}\right)$.

Наконец, инвариантность $\mathscr{Q}^{u v}$ относительно $Z$ следует из того, что при изоморфизме $\Lambda^{u v}$ оператор $Z^{1 u v}$ переходит в $Z$ :

$$
Z \Lambda^{u v}=\Lambda^{u v} Z^{1 u v}
$$

а подпространство $\mathscr{P}^{1 u v}$ инвариантно относительно $Z^{1 u v}$. Предложение доказано.

Мы показали, что на каждом подпространстве $\mathscr{Q}^{u v} \subset \mathscr{A}$ функций сбалансированного роста оператор умножения на $\mathbf{i}$ определяется канонически с точностью до знака требованием коммутирования с базисными сдвигами. Имеются непрерывные семейства функций, параметры $(u, v)$ которых меняются произвольным образом в пределах области допустимых значений, причем эта область стягиваема. Поэтому, выбрав знак для оператора умножения на $\mathbf{i}$ в одном из $\mathscr{Q}^{u v}$, мы можем однозначно продолжить этот выбор на остальные подпространства из соображений непрерывности.

Таким образом, доказана следующая теорема.

Tеорема 5. B подпространстве

$$
\mathscr{Q}=\bigoplus_{u, v} \mathscr{Q}^{u v} \subset \mathscr{A}
$$

порожденном всеми дискретными аналитическими функииями сбалансированного роста, существует, и притом единственный с точностью до знака, оператор I, обладающий свойствами:

1) I коммутирует с базисными сдвигами решетки;

2) I сохраняет каждое из подпространств $\mathscr{Q}^{u v}$;

3) если $f_{t}(m, n)$ - непрерьвно зависящая от параметра $t$ функция сбалансированного роста, то $\Im f_{t}$ также непрерывно зависит от $t$.

Oператоры $D=T_{1}+T_{1}+T_{1} T_{2}$ u $Z=m T_{2}-n T_{1}$ сохраняют подпространство $\mathscr{Q}$ и являются $\mathbb{C}$-линейными на нем, если умножение на $\mathbf{i}$ задается оператором $\mathfrak{I}$.

Совокупность функиий вида

$$
\varphi_{k}(m, n ; u, v)=\left.u^{m} v^{n} \operatorname{Re}\left(\frac{\partial^{k}}{\partial \kappa^{k}} E^{1 u v}(m, n ; \kappa)\right)\right|_{\kappa=0},
$$


где $k \geqslant 0$, тройка $(1, u, v)$ удовлетворяет неравенствам треугольника, а $E^{1 u v}$ задается формулой (17), является базисом пространства $\mathscr{Q}$ над $\mathbb{C}$, причем

$$
\begin{aligned}
& D \varphi_{k}(m, n ; u, v)=k \varphi_{k-1}(m, n ; u, v), \\
& Z \varphi_{k}(m, n ; u, v)=\sum_{i=0}^{k+1} a_{k i} \varphi_{i}(m, n ; u, v), \quad a_{k, k+1} \neq 0,
\end{aligned}
$$

где $a_{i j}$ - некоторые комплексные коэфбициенты, умножение на которые понимается в смысле комплексной структуры, заданной оператором $\mathfrak{~}$

ЗАмЕчАНИЕ 2. Функции сбалансированного роста по определению имеют вид $g(m, n) e^{\ell(m, n)}$, где $g(m, n)$ - функция, ограниченная многочленом, a $\ell(m, n)$ линейная функция. При этом допустимые значения для коэффициентов функции $\ell$ пробегают внутренность области, которая в терминологии, предложенной в [24], называется амебой, отвечающей многочлену $1+z+w$ (см. рис. 2).

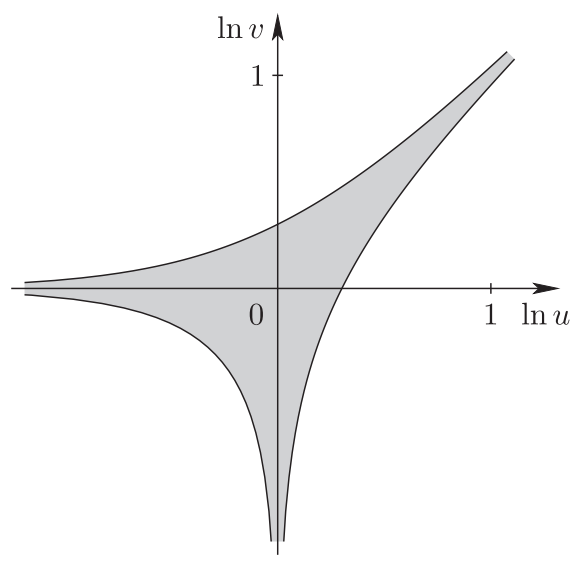

Рис. 2. Область допустимых значений для параметров $u, v$ функции сбалансированного роста

Класс функций сбалансированного роста инвариантен относительно поворота решетки $\mathbb{L}_{\triangle}$ на $2 \pi / 3$, но не инвариантен относительно поворота на $\pi / 3$.

\section{4. Четырехугольная дискретизация на ромбической решетке}

Для четырехугольной дискретизации (4) условий Коши-Римана вещественная калибровка, аналогичная (14), возможна, только если параметр решетки $\tau$ равен единице по абсолютной величине. Такие решетки называются ромбическими, так как фундаментальная ячейка имеет вид ромба.

В случае $|\tau|=1$ величина $(\tau+1) /(\tau-1)$ чисто мнимая. Введем обозначение

$$
\delta=\mathbf{i} \frac{\tau+1}{\tau-1} \in \mathbb{R} .
$$


Через $\mathbb{L}^{\delta}$ будем обозначать соответствующую ромбическую решетку. Уравнение (4) запишется в виде

$$
f(m, n)+\mathbf{i} \delta f(m+1, n)-\mathbf{i} \delta f(m, n+1)-f(m+1, n+1)=0 .
$$

Сделав калибровочное преобразование $f(m, n) \mapsto \mathbf{i}^{m-n} f$, мы приходим к уравнению с вещественными коэффициентами:

$$
f(m, n)+\delta f(m+1, n)+\delta f(m, n+1)-f(m+1, n+1)=0 .
$$

ОПрЕДЕЛЕниЕ 4. Вещественнозначная функция на $\mathbb{L}^{\delta}$ называется $\partial и с к р е т-$ ной голоморфной функиией четырехугольного типа, если она является решением разностного уравнения (19).

Далее речь пойдет только о четырехугольном типе дискретных голоморфных функций, поэтому указание типа мы опускаем. Аналогами операторов Коши-Римана и дифференцирования для функций на $\mathbb{L}^{\delta}$ будут

$$
Q^{\delta}=1+\delta\left(T_{1}+T_{2}\right)-T_{1} T_{2} \quad \text { и } \quad D^{\delta}=-1+\delta\left(T_{1}+T_{2}\right)+T_{1} T_{2}=T_{1} T_{2}\left(Q^{\delta}\right)^{+}
$$

соответственно. В качестве аналога оператора $Z$ мы возьмем

$$
Z^{\delta}=m\left(\delta T_{1}+1\right)-n\left(\delta T_{2}+1\right) .
$$

Этот оператор не коммутирует с $Q^{\delta}$, как было в случае треугольной дискретизации, но выполнено соотношение

$$
Q^{\delta} Z^{\delta}=\left(Z^{\delta}+\delta\left(T_{1}-T_{2}\right)\right) Q^{\delta}
$$

из которого следует, что $Z^{\delta}$ сохраняет пространство $\mathscr{A}^{\delta}$ дискретных голоморфных функций на $\mathbb{L}^{\delta}$.

В отличие от треугольного случая операторы $\Lambda^{u v}$ не задают теперь изоморфизмы между пространствами дискретных голоморфных функций на ромбических решетках с разными геометриями (разными $\delta$ ). Однако для каждой решетки $\mathbb{L}^{\delta}$ имеет место аналог теоремы 5 , который мы сейчас сформулируем.

ОПРЕДЕЛЕНИЕ 5. Функция $f$ на $\mathbb{L}^{\delta}$ будет называться функиией $\delta$-сбалансированного роста, если найдутся $k \in \mathbb{N}$ и $C_{1}, C_{2}, u, v>0$ такие, что

1) существует четырехугольник с длинами сторон $1, u \delta, v \delta, u v$;

2) для всех $(m, n) \in \mathbb{L}^{\delta}$ выполнено неравенство

$$
f(m, n)<C_{1}+C_{2}\left(m^{2}+n^{2}\right)^{k / 2} u^{m} v^{n} .
$$

Пространство $\delta$-сбалансированных дискретных аналитических функций на $\mathbb{L}^{\delta}$, удовлетворяющих (20) для фиксированных $u$ и $v$, будем обозначать через $\mathscr{Q}^{\delta u v}$.

ЗАмечАниЕ 3. Множество допустимых значений для параметров $\log u, \log v$ функции $\delta$-сбалансированного роста образует внутренность амебы, соответствующей многочлену $1+\delta(z+w)-z w$ (см. рис. 3$)$.

ТЕОРема 6. При каждом фиксированном $\delta>0$ подпространства $\mathscr{Q}^{\delta и v}$ функций сбалансированного роста при всевозможных допустимых парах $(u, v)$ образуют прямую сумму в пространстве $\mathscr{A}^{\delta}$. 


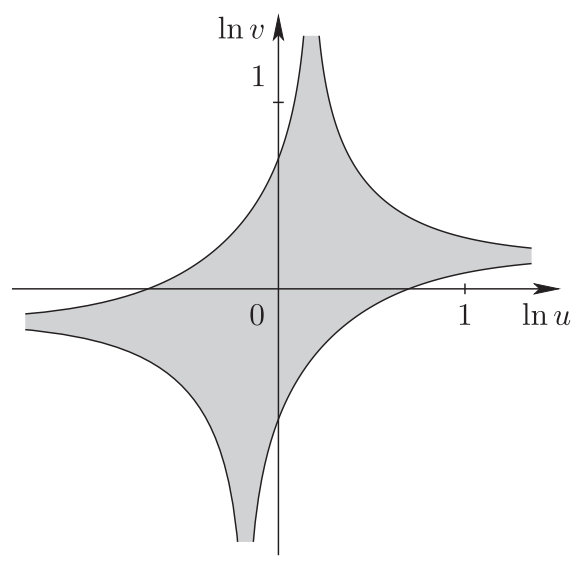

Рис. 3. Область допустимых значений для параметров $u, v$ функции $\delta$-сбалансированного роста при $\delta=1.5$

В подпространстве

$$
\mathscr{Q}^{\delta}=\bigoplus_{u, v} \mathscr{Q}^{\delta u v} \subset \mathscr{A}^{\delta}
$$

порожденном всеми дискретнъми аналитическими функииями $\delta$-сбалансированного роста, существует, и притом единственный с точностью до знака, оператор $\mathfrak{I}^{\delta}$, обладающий свойствами:

1) $\mathfrak{I}^{\delta}$ коммутирует с базисными сдвигами решетки;

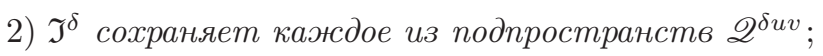

3) если $f_{t}(m, n)$ - непрерывно зависящая от параметра $t$ функиия $\delta$-сбалансированного роста, то $\mathfrak{I}^{\delta} f_{t}$ также непрерьвно зависит от $t$.

Oператори $D^{\delta}=-1+\delta\left(T_{1}+T_{2}\right)+T_{1} T_{2} u Z^{\delta}=m\left(\delta T_{1}+1\right)-n\left(\delta T_{2}+1\right)$ сохраняют подпространство $\mathscr{Q}^{\delta}$ и являются $\mathbb{C}$-линейными на нем, если умножение на і задается оператором $\mathfrak{I}^{\delta}$.

Совокупность функций вида

$$
\psi_{k}(m, n ; \delta, u, v)=\left.u^{m} v^{n} \operatorname{Re}\left(\frac{\partial^{k}}{\partial \kappa^{k}} \lambda(\kappa ; \delta, u, v)^{m} \mu(\kappa ; \delta, u, v)^{n}\right)\right|_{\kappa=0},
$$

где $k \geqslant 0,(1, \delta u, \delta v, u v)$ - набор длин сторон некоторого четьрехугольни$\kappa a$, а $\lambda(\kappa ; \delta, u, v), \mu(\kappa ; \delta, u, v)$ - аналитические функиии в окрестности точки $\kappa=0$, определяемые из системы

$$
\left\{\begin{array}{l}
1+\delta(u \lambda+v \mu)-u v \lambda \mu=0 \\
-u v+\delta(v \lambda+u \mu)+\lambda \mu=\kappa \\
\operatorname{Im} \lambda(0 ; \delta, u, v)>0
\end{array}\right.
$$


является базисом пространства $\mathscr{Q}^{\delta}$ над $\mathbb{C}$, причем

$$
\begin{aligned}
& D \psi_{k}(m, n ; \delta, u, v)=k \psi_{k-1}(m, n ; \delta, u, v), \\
& Z \psi_{k}(m, n ; \delta, u, v)=\sum_{i=0}^{k+1} b_{k i} \psi_{i}(m, n ; \delta, u, v), \quad b_{k, k+1} \neq 0,
\end{aligned}
$$

где $b_{i j}=b_{i j}(\delta, u, v)$ - некоторые комплексные коэффициенты, умножение на которые понимается в смысле комплексной структуры, заданной оператором $\mathfrak{I}^{\delta}$.

ДокАЗАтельство. Рассуждение полностью следует схеме доказательства аналогичных утверждений для треугольной дискретизации. Мы напомним кратко основные этапы и дадим явные формулы для использующихся в доказательстве операторов и функций, которые отличаются от треугольного случая.

Пусть $a, b, c, d$ - длины сторон некоторого четырехугольника на евклидовой плоскости. Тогда найдется с точностью до движения ровно один четырехугольник с длинами сторон $a, b, d, c$ (в этом порядке), вписанный в окружность.

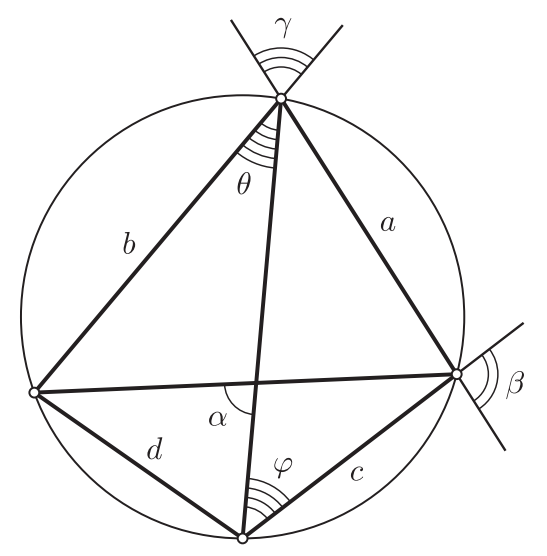

Рис. 4. Связь между параметрами $a, b, c, d, \alpha, \beta, \gamma, \varphi, \theta$

Обозначим через $\alpha$ угол между диагоналями такого четырехугольника (любой из двух), через $\beta$ и $\gamma$ углы $\angle a c$ и $\angle a b$ соответственно, а через $\varphi(\theta)-$ угловой размер стороны $a$ (соответственно $d$ ) для любого из концов противоположной стороны (см. рис. 4). Введем также обозначение

$$
\sigma=\frac{\sin \varphi \sin \theta}{2 \sin \alpha \sin \beta \sin \gamma}
$$

Обозначим через $\mathscr{P}_{k}^{a b c d}$ для каждого $k=0,1,2, \ldots$ пространство решений системы разностных уравнений

$$
Q^{a b c d} f=0, \quad\left(D^{a b c d}\right)^{k+1} f=0,
$$

где

$$
Q^{a b c d}=1+\frac{b}{a} T_{1}+\frac{c}{a} T_{2}-\frac{d}{a} T_{1} T_{2}, \quad D^{a b c d}=-\frac{d}{a}+\frac{c}{a} T_{1}+\frac{b}{a} T_{2}+T_{1} T_{2},
$$


через $\mathscr{P}^{a b c d}$ объединение этих пространств по всем $k$, а через $\mathscr{A}^{a b c d}$ - пространство всех решений уравнения $Q^{a b c d} f=0$.

В данном случае мы не интерпретируем $\mathscr{A}^{a b c d}$ и $\mathscr{P}^{a b c d}$ как пространства дискретных голоморфных функций или многочленов на некоторой решетке, но можем проделать с ними то же, что с аналогичными пространствами в случае треугольной дискретизации.

А именно, так же, как в треугольном случае, можно показать, что для всех $k \geqslant 0$ пространство $\mathscr{P}_{k}^{a b c d}$ имеет над $\mathbb{R}$ размерность $2 k+2$. Для этого устанавливаются два факта:

1) пространство $\mathscr{P}_{0}^{a b c d}=\operatorname{ker} Q^{a b c d} \cap \operatorname{ker} D^{a b c d}$ двумерно;

2) система уравнений $Q^{a b c d} f=0, D^{a b c d} f=g$ разрешима относительно $f$ тогда и только тогда, когда $Q^{a b c d} g=0$.

В построении оператора комплексной структуры $\mathfrak{I}^{a b c d}$ используется тождество

$$
\begin{aligned}
\left(T_{2}+\cos \beta-\sigma(\sin \beta) D^{a b c d}\right)^{2}=- & \sin ^{2} \beta\left(1+2 \sigma(\operatorname{ctg} \theta) D^{a b c d}-\left(\sigma D^{a b c d}\right)^{2}\right) \\
& +\frac{\sin \varphi}{\sin \alpha \sin \gamma}\left((\sin \varphi) T_{2}+\sin (\beta+\varphi)\right) Q^{a b c d}
\end{aligned}
$$

которое позволяет определить $\mathfrak{I}^{a b c d}$ на $\mathscr{P}^{a b c d}$ формулой

$$
\mathfrak{I}^{a b c d}=\frac{1}{\sin \beta}\left(T_{2}+\cos \beta-\sigma(\sin \beta) D^{a b c d}\right)\left(1+2 \sigma(\operatorname{ctg} \theta) D^{a b c d}-\left(\sigma D^{a b c d}\right)^{2}\right)^{-1 / 2} .
$$

Эту формулу следует понимать так же, как в треугольном случае: нужно сначала разложить правую часть в ряд по $D^{a b c d}$, а затем при ограничении на каждое $\mathscr{P}_{k}^{a b c d}$ отбросить члены порядка больше $k$.

Если $\beta+\gamma \neq \pi$, то оператор $\mathfrak{I}^{a b c d}$ можно записать в более симметричном виде:

$$
\begin{aligned}
\mathfrak{I}^{a b c d}= & \frac{1}{\sin (\beta+\gamma)}\left((\cos \gamma) T_{2}-(\cos \beta) T_{1}+\sigma(\sin (\gamma-\beta)) D^{a b c d}\right) \\
& \times\left(1+2 \sigma(\operatorname{ctg} \theta) D^{a b c d}-\left(\sigma D^{a b c d}\right)^{2}\right)^{-1 / 2} .
\end{aligned}
$$

Доказательство единственности для $\mathfrak{I}^{a b c d}$ дословно повторяет аналогичное рассуждение для треугольного случая.

Для построения базиса в $\mathscr{P} a b c d$ используется экспоненциальная функция $E^{a b c d}$, которая является решением системы $Q^{a b c d} E^{a b c d}=0, D^{a b c d} E^{a b c d}=\kappa E^{a b c d}$. Ее явный вид (определяемый однозначно с точностью до комплексного сопряжения и умножения на константу) таков:

$$
\begin{aligned}
E^{a b c d}(m, n ; \kappa)=( & \left.-\cos \gamma+\sigma \kappa \sin \gamma+\mathbf{i} \sin \gamma \sqrt{1+2 \sigma \kappa \operatorname{ctg} \theta-(\sigma \kappa)^{2}}\right)^{m} \\
& \times\left(-\cos \beta+\sigma \kappa \sin \beta-\mathbf{i} \sin \beta \sqrt{1+2 \sigma \kappa \operatorname{ctg} \theta-(\sigma \kappa)^{2}}\right)^{n} .
\end{aligned}
$$


Для доказательства того, что $\mathscr{P}^{a b c d}$ содержит все функции из $\mathscr{A}^{a b c d}$ полиномиального роста, нужны формула Коши и убывающая на бесконечности функция Грина для $Q^{a b c d}$. Формула Коши пишется так же, как в треугольном случае (см. (12)), с той лишь разницей, что сумма теперь берется по всем $\left(m^{\prime}, n^{\prime}\right)$, для которых ромб с вершинами $\left(m^{\prime}, n^{\prime}\right),\left(m^{\prime}+1, n^{\prime}\right),\left(m^{\prime}+1, n^{\prime}+1\right)$, $\left(m^{\prime}, n^{\prime}+1\right)$ пересекает $\partial \Omega$.

Функция Грина для $Q^{a b c d}$ строится классическим образом:

$$
G^{a b c d}(m, n)=\frac{1}{4 \pi^{2}} \int_{0}^{2 \pi} \int_{0}^{2 \pi} \frac{e^{\mathbf{i}(m x+n y)}}{1+(b / a) e^{\mathbf{i} x}+(c / a) e^{\mathbf{i} y}-(d / a) e^{\mathbf{i}(x+y)}} d x d y .
$$

Для любого $k=0,1,2, \ldots$ функция $\left(Q^{a b c d}\right)^{k} G^{a b c d}$ убывает на бесконечности как $o\left(\left(m^{2}+n^{2}\right)^{-k / 2}\right)$. Это следует из того, что подынтегральная функция в

$$
\begin{aligned}
& \left(Q^{a b c d}\right)^{k} G^{a b c d}(m, n ; \kappa) \\
& \quad=\frac{1}{4 \pi^{2}} \int_{0}^{2 \pi} \int_{0}^{2 \pi} \frac{e^{\mathbf{i}(m x+n y)}\left(-(d / a)+(c / a) e^{\mathbf{i} x}+(b / a) e^{\mathbf{i} y}+e^{\mathbf{i}(x+y)}\right)^{k}}{1+(b / a) e^{\mathbf{i} x}+(c / a) e^{\mathbf{i} y}-(d / a) e^{\mathbf{i}(x+y)}} d x d y
\end{aligned}
$$

имеет класс гладкости $C^{(k-1)}$ при $k>0$ и интегрируема при $k=0$.

Наконец, заметим, что имеет место равенство

$$
Q^{\delta} \Lambda^{u v}=\Lambda^{u v} Q^{a b c d}, \quad \text { где } \quad a=1, \quad b=u \delta, \quad c=v \delta, \quad d=u v,
$$

из которого следует, что $\mathscr{Q}^{\delta u v}=\Lambda^{u v}\left(Q^{1, u \delta, v \delta, u v}\right)$.

Дальнейшие рассуждения не содержат ничего нового по сравнению с треугольным случаем.

\section{Список литературы}

[1] I. A. Dynnikov, S. P. Novikov, "Geometry of the triangle equation on two-manifolds", Mosc. Math. J., 3:2 (2003), 419-438.

[2] S. P. Novikov, "Difference analogs of Laplace transformations and two-dimensional Toda lattices", Appendix I in: S.P. Novikov, A.P. Veselov "Exactly solvable 2-dimensional Schrödinger operators and Laplace transformations", Solitons, geometry and topology: on the crossroads, Amer. Math. Soc. Transl. Ser. 2, 179, eds. V. M. Buchstaber, S. P. Novikov, Amer. Math. Soc., Providence, RI, 1997, $124-126$.

[3] С. П. Новиков, "Алгебраические свойства двумерных разностных операторов", УМH, 52:1(313) (1997), 225-226; англ. пер.: S. P. Novikov, "Algebraic properties of two-dimensional difference operators", Russian Math. Surveys, 52:1 (1997), 226-227.

[4] И.А. Дынников, С.П. Новиков, "Преобразования Лапласа и симплициальные связности", УМН, 52:6(318) (1997), 157-158; англ. пер.: I. A. Dynnikov, S. P. Novikov, "Laplace transforms and simplicial connections", Russian Math. Surveys, 52:6 (1997), 1294-1295.

[5] С. П. Новиков, И. А. Дынников, “Дискретные спектральные симметрии маломерных дифференциальных операторов и разностных операторов на правильных решетках и двумерных многообразиях", УМH, 52:5(317) (1997), 175-234; англ. пер.: S. P. Novikov, I. A. Dynnikov, "Discrete spectral symmetries of low-dimensional differential operators and difference operators on regular lattices and two-dimensional manifolds", Russian Math. Surveys, 52:5 (1997), 1057-1116. 
[6] J. Ferrand, "Fonctions préharmoniques et fonctions préholomorphes", Bull. Sci. Math. (2), 68 (1944), 152-180.

[7] R. P. Isaacs, "A finite difference function theory", Univ. Nac. Tucumán. Revista A, 2 (1941), 177-201.

[8] R. J. Duffin, "Basic properties of discrete analytic functions", Duke Math. J., 23:2 (1956), 335-363.

[9] Ch. Mercat, "Discrete Riemann surfaces and the Ising model", Comm. Math. Phys., 218:1 (2001), 177-216.

[10] S. Smirnov, "Discrete complex analysis and probability", Proceedings of the International Congress of Mathematicians 2010 (ICM 2010), v. I (Hyderabad, India, August 19-27, 2010), Hindustan Book Agency, New Delhi, 2011, 595-621.

[11] R. J. Duffin, "Potential theory on a rhombic lattice", J. Combin. Theory, 5:3 (1968), $258-272$.

[12] R. Kenyon, "The Laplacian and Dirac operators on critical planar graphs", Invent. Math., 150:2 (2002), 409-439.

[13] R. Kenyon, J.-M. Schlenker, "Rhombic embeddings of planar quad-graphs", Trans. Amer. Math. Soc., 357:9 (2005), 3443-3458.

[14] Ch. Mercat, "Discrete Riemann surfaces", Handbook of Teichmüller theory, v. I, IRMA Lect. Math. Theor. Phys., 11, Eur. Math. Soc., Zürich, 2007, 541-575.

[15] D. Chelkak, S. Smirnov, "Discrete complex analysis on isoradial graphs", Adv. Math., 228:3 (2011), 1590-1630.

[16] A. Bobenko, M. Skopenkov, "Discrete Riemann surfaces: linear discretization and its convergence", 2012 (v2 - 2013), 27 pp., arXiv: 1210.0561.

[17] Н.П. Долбилин, М.А. Штанько, М.И. Штогрин, "Кубические подкомплексы в правильных решетках", Докл. АН СССР, 291:2 (1986), 277-279; англ. пер.: N.P. Dolbilin, M. A. Shtan'ko, M. I. Shtogrin, "Cubic subcomplexes in regular lattices", Soviet Math. Dokl., 34:3 (1987), 467-469.

[18] Н. П. Долбилин, М. А. Штанько, М. И. Штогрин, "Квадрильяжи и параметризации решетчатых циклов", Топология и ее приложения, Труды Международной топологической конференции (Баку, 3-8 октября 1987 г.), Тр. МИАН СССР, 193, Наука, M., 1992, 108-114; англ. пер.: N. P. Dolbilin, M. A. Shtan'ko, M. I. Shtogrin, "Quadrillages and parametrizations of lattice cycles", Proc. Steklov Inst. Math., 193 (1993), 117-123.

[19] L. Lusternik, "Über einige Anwendungen der direkten Methoden in Variationsrechnung”, Матем. сб., 33:2 (1926), 173-201.

[20] W.P. Thurston, "Zippers and univalent functions", The Bieberbach conjecture (West Lafayette, IN, 1985), Math. Surveys Monogr., 21, Amer. Math. Soc., Providence, RI, 1986, 185-197.

[21] A. Bobenko, Ch. Mercat, Yu. B. Suris, "Linear and nonlinear theories of discrete analytic functions. Integrable structure and isomonodromic Green's function", J. Reine Angew. Math., 2005:583 (2005), 117-161.

[22] S. P. Novikov, "New discretization of complex analysis: the Euclidean and hyperbolic planes", Современные проблемы математики, Сборник статей. K 75-летию Института, Тр. МИАН, 273, МАИК, М., 2011, 257-270; англ. пер.: S. P. Novikov, "New discretization of complex analysis: the Euclidean and hyperbolic planes", Proc. Steklov Inst. Math., 273 (2011), 238-251. 
[23] П.Г. Гриневич, Р. Г. Новиков, "Ядро Коши для DN-дискретного комплексного анализа Новикова-Дынникова на треугольной решетке”, УМН, 62:4(376) (2007), 155-156; англ. пер.: P. G. Grinevich, R. G. Novikov, "The Cauchy kernel for the Novikov-Dynnikov DN-discrete complex analysis in triangular lattices", Russian Math. Surveys, 62:4 (2007), 799-801.

[24] I. M. Gelfand, M. M. Kapranov, A. V. Zelevinsky, Discriminants, resultants, and multidimensional determinants, Math. Theory Appl., Birkhäuser Boston, Inc., Boston, MA, 1994, x+523 pp.

Иван Алексеевич Дынников

Поступила в редакцию

(Ivan A. Dynnikov)

Математический институт им. В. А. Стеклова

Российской академии наук

E-mail: dynnikov@mech.math.msu.su 